\title{
Effects of Two Different Pozole Preparation Processes on Quality Variables and Pasting Properties of Processed Maize Grain
}

\author{
María Gricelda Vázquez-Carrillo, ${ }^{1}$ David Santiago-Ramos, ${ }^{2}$ \\ Edith Domínguez-Rendón, ${ }^{1}$ and Marco Antonio Audelo-Benites ${ }^{1}$ \\ ${ }^{1}$ Campo Experimental Valle de México, Instituto Nacional de Investigaciones Forestales Agrícolas y Pecuarias (INIFAP), \\ Km. 13.5 Carretera Los Reyes-Texcoco, Coatlinchán, 56250 Texcoco, MEX, Mexico \\ ${ }^{2}$ Programa de Posgrado en Alimentos del Centro de la República (PROPAC), Facultad de Química, \\ Universidad Autónoma de Querétaro, Cerro de las Campanas S/N, Col. Las Campanas, 76010 Querétaro, QRO, Mexico \\ Correspondence should be addressed to María Gricelda Vázquez-Carrillo; gricelda_vazquez@yahoo.com
}

Received 6 July 2016; Revised 26 September 2016; Accepted 23 October 2016; Published 12 January 2017

Academic Editor: Alejandro Hernández

Copyright (C) 2017 María Gricelda Vázquez-Carrillo et al. This is an open access article distributed under the Creative Commons Attribution License, which permits unrestricted use, distribution, and reproduction in any medium, provided the original work is properly cited.

\begin{abstract}
The effects of two different pozole preparation processes, traditional (TP) and industrial (IP), on quality variables, chemical composition, and pasting properties of processed grain of nine maize landraces were evaluated. Nixtamalization and steeping time in TP $(\sim 15 \mathrm{~h})$ allowed more water absorption resulting in higher moisture content as well as softer debranned nixtamal relative to the debranned nixtamal produced by IP (52 min). Steeping in TP and bleaching in IP increased the pasting temperature, peak viscosity, and time to peak viscosity of maize starch. Flowering time was shorter in IP $(<120 \mathrm{~min})$ than in TP $(>120 \mathrm{~min})$ and was significantly affected by the hardness of debranned nixtamal and bleached precooked grains. Total dry matter loss was higher in IP $(>10.5 \%)$ than in TP $(<5.0 \%)$, mainly due to the complete elimination of pedicel and pericarp by the $\mathrm{Ca}(\mathrm{OH})_{2}+\mathrm{NaOH}$ solution during cooking. Soft grains, with low test weight, a high proportion of floury endosperm, and high peak viscosity, are required to obtain higher yield of bleached precooked grains and soft flowered grains in both processes.
\end{abstract}

\section{Introduction}

Pozole is a pre-Columbian dish widely consumed in Mexico and in the United States by people of Latin American origin [1]. The production of maize for pozole preparation and the consumption of this dish have increased in recent years [2].

The main ingredient of pozole is boiled maize in the form of "flowered grain." There are two different processes involved in obtaining flowered grains for pozole: the traditional process (TP) and the commercial or industrial process (IP) [3]. Both processes begin with nixtamalization, a thermal-alkaline treatment whose principal purpose is to remove the pedicel and pericarp from the grain. In TP, nixtamalization is carried out with $\mathrm{Ca}(\mathrm{OH})_{2}$, which hydrolyzes the pericarp, while, in IP, a mixture of $\mathrm{Ca}(\mathrm{OH})_{2}$ and $\mathrm{NaOH}$ is used to remove both the pedicel and pericarp. In TP, the cooked grain (nixtamal) is steeped for $8-16 \mathrm{~h}$. It is then washed to eliminate the pericarp as well as the pedicel (deheaded), and finally the debranned nixtamal is boiled, causing the grain to swell and open up into a flower shape (flowered grain) [1, 3-6]. In IP, the debranned nixtamal is washed and the pedicel is removed immediately (deheaded). The grains are then bleached in a solution of sodium metabisulfite $\left(\mathrm{Na}_{2} \mathrm{~S}_{2} \mathrm{O}_{5}\right)$ and acetic acid $\left(\mathrm{CH}_{3} \mathrm{COOH}\right)$ and packed, obtaining a product ready to be cooked (bleached precooked grain) until it flowers when used by the end consumer $[2,3]$.

In Mexico, about sixteen maize landraces are used in the preparation of flowered grains for pozole [2, 7-10]. Many of these landraces, including Cacahuacintle, Ancho, Elotes Occidentales, and Jala, are known as "specialty maize landraces," which means they are preferred over hybrids or 
improved varieties for the preparation of specific food or dishes like pozole $[2,8,10,11]$.

Bonifacio Vázquez et al. [4] studied the relationship between physical grain characteristics of the Cacahuacintle landrace and the quality of the flowered grain for pozole prepared by TP. They found that the shape, size, and density of the grain affect flowering time and flowered grain quality. The larger and globose-shaped grains require less flowering time and result in a better quality product. Figueroa et al. [1] reported that the peak viscosity of the grain is a good parameter in predicting flowered grain yield by TP, due to the significant correlation found between those two variables $(r=0.73, p<0.001)$. On the other hand, Figueroa et al. $[1,5]$ reported that the annealing process is carried out in the steeping step of TP. This phenomenon has two important implications: (1) an increase in the gelatinization temperature and pasting temperature of the nixtamal starch, which increases the stability of the starch granules and their ability to resist collapse during boiling, and (2) an increase in viscosity, swelling, and the hydration capacity of the starch granules, resulting in a soft texture and a high yield of pozole $[1,5]$.

Industrial processing has greater economic and technological implications. Vázquez-Carrillo and Santiago-Ramos [3] reported that IP reduces processing time and energy consumption considerably, and it may also reduce nutritional quality. However, the effect of this process on the quality variables of the resulting products and on the properties of starch and other grain components is still unknown.

Keleman and Hellin [8] and Hellin et al. [10] noted that research on maize landraces has focused on yield and agronomic features while aspects of grain quality and culinary potential have been given less attention, so it is necessary to expand the scope of research and determine the potential of each maize landrace for specialty markets in this case, the best landraces for pozole, which could generate more income for farmers and prevent the extinction of some landraces through "conservation in situ" or "conservation through use." Regarding processors, some industries that produce bleached precooked grain also opt to use hybrids, varieties, and landraces grown commonly for making tortillas such as Tuxpeño, Cónico or Chalqueño, due to their low price and wide availability, and then mix them with the other landraces. However, the effect of this process on these types of maize and how it affects the quality of pozole are unknown.

The objective of this study was to evaluate the effects of two different pozole preparation processes, industrial and traditional, on the quality variables and pasting properties of the debranned nixtamal, bleached precooked grain, and flowered grain of maize.

\section{Materials and Methods}

2.1. Materials. Nine maize landraces were assessed. Eight landraces are well documented as being used in the preparation of pozole: Cacahuacintle, Ancho, Tabloncillo, Jala, BofoHarinoso de Ocho, Bolita, Chalqueño, and Tuxpeño [2, 710]. Cónico was used for purposes of comparison. Tuxpeño and Cónico are landraces such that their main use is the preparation of tortillas, but, as mentioned in the Introduction, some industries that produce bleached precooked grain also opt to use these types of maize due to their low price and wide availability. The samples correspond to a collection of each landrace harvested in 2011 in the following locations: Cacahuacintle from Topilejo, Ciudad de Mexico; Ancho from Iguala, Guerrero; Tabloncillo from Obregón, Sonora; Jala from Jala, Nayarit; Bofo-Harinoso de Ocho from El Roble, Nayarit; Bolita from Etla, Oaxaca; Chalqueño from Ayapango, Estate of Mexico; Tuxpeño from Sinaloa; and Cónico from Los Reyes, Tlaxcala. Two commercial samples of bleached precooked grain were used as controls, and these were obtained at the local market.

2.2. Physical Grain Characteristics. Test weight (TW) was determined by weighing a fixed volume $(1 \mathrm{~L})$ of grain from a test weight filling hopper (Ohaus ${ }^{\circledR}$ Model 150, Florham Park, NJ, USA) according to the 55-10.01 AACC International Method [12]. Hundred-grain weight (HGW) was measured by weighing 100 grains in an analytical balance Ohaus Model TS400S (Florham Park, NJ, USA); this variable was used as an indirect measure of grain size. Flotation index (FI) was determined with the method described in the norm NMX-FF034/1-SCFI-2002 [13], which consists of placing 100 grains in a beaker containing $300 \mathrm{~mL}$ of $\mathrm{NaNO}_{3}$ solution $(1.250 \mathrm{~g} / \mathrm{mL})$, grains were stirred and left standing for $1 \mathrm{~min}$, and finally the number of floating kernels was recorded. TW, HGW, and FI are affected by the moisture content of the sample, so they were adjusted at $12 \%$ moisture content by using the following equations:

$$
\begin{aligned}
& \text { Adjusted TW } \\
& \begin{aligned}
= & \frac{100-12 \%}{100-\text { Sample moisture content }(\%)} \\
& \times \text { Measured TW, }
\end{aligned}
\end{aligned}
$$

Adjusted HGW

$$
\begin{aligned}
= & \frac{100-12 \%}{100-\text { Sample moisture content }(\%)} \\
& \times \text { Measured HGW }
\end{aligned}
$$

Adjusted FI

$$
\begin{aligned}
= & \frac{100-12 \%}{100-\text { Sample moisture content }(\%)} \\
& \times \text { Measured FI. }
\end{aligned}
$$

Moisture content was determined by the dielectric meter method 44-11.01 of the AACC International [12] using $250 \mathrm{~g}$ in a Steinlite Moisture Tester SS250 (Atchison, KS, USA).

Percentages of pedicel, pericarp, germ, floury endosperm, and vitreous endosperm were determined by hand-dissection. Twenty-five grains were soaked in $50 \mathrm{~mL}$ of distilled water at $60^{\circ} \mathrm{C}$ for $30 \mathrm{~min}$, and then with a scalpel each structure was separated. Grain fractions were dried for $12 \mathrm{~h}$ in an oven at $100^{\circ} \mathrm{C}$ to express the percentage in dry basis [5]. 


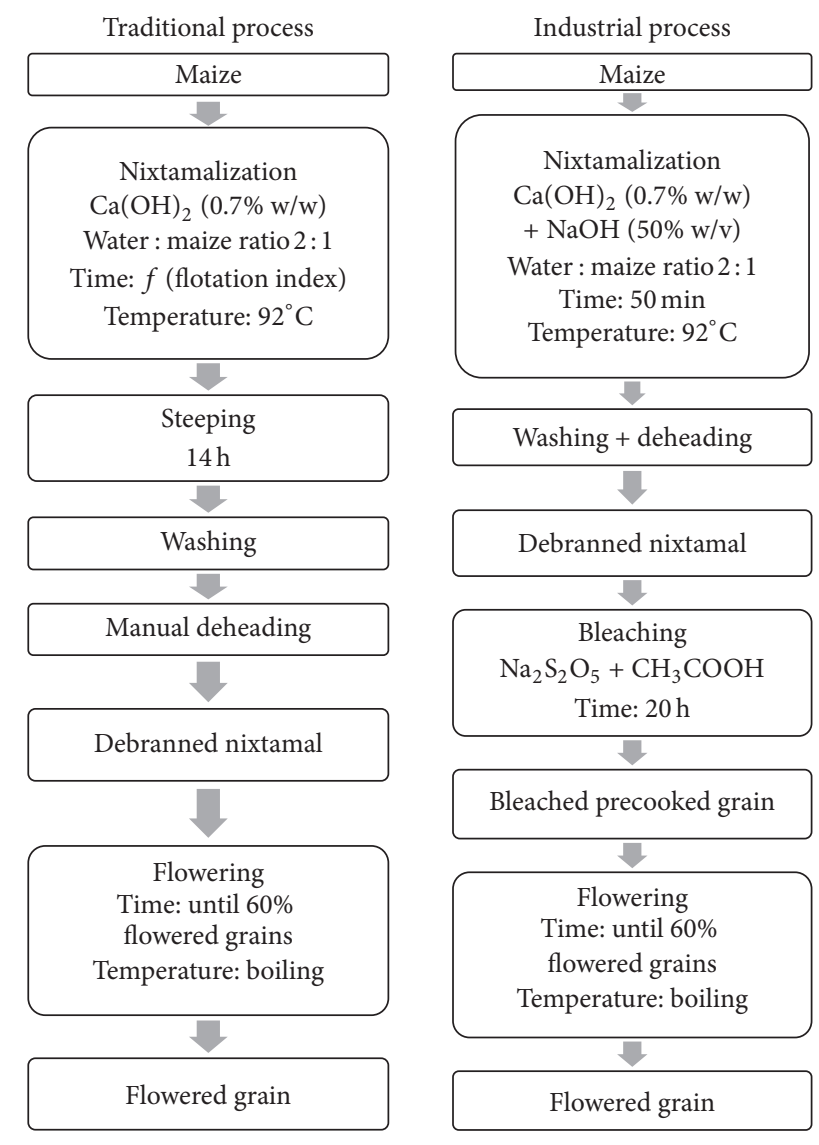

FIGURE 1: Summarized outlines of traditional and industrial processes to obtain flowered grain for pozole preparation.

Pericarp thickness (PT) was measured following the method described by Wolf et al. [14]. Five kernels were steeped in water for $4 \mathrm{~h}$ at room temperature, and then crown cap and tip cap were cut and removed. Pericarp of each grain was peeled as strips and placed at room temperature for $24 \mathrm{~h}$ to dry. Pericarp thickness was measured with a micrometer at the position opposite to germ and was reported as the mean of five strips in $\mu \mathrm{m}$.

Grain luminosity $\left(L^{*}\right)$ was determined with the colorimeter Hunter Lab MiniScan XE Plus Model 45/0-L ${ }^{\circledR}$ (Reston, VA, USA), with a D/65 illuminant and a $10^{\circ}$ angle. The colorimeter was calibrated with a standard white tile and samples were placed randomly in a $5.5 \mathrm{~cm}$ diameter plastic cell with an optically clear glass bottom, according to the method reported by Floyd et al. [15].

All variables were analyzed in duplicate.

\subsection{Samples Preparation: Traditional and Industrial Processes.} Two batches of each sample were run. Two different processes were used to obtain flowered grains for pozole preparation: the traditional process (TP) and the industrial process (IP) (Figure 1), based on the methodology described by VázquezCarrillo and Santiago-Ramos [3].
2.3.1. Traditional Process. For nixtamalization, $100 \mathrm{~g}$ of clean, uniform-size maize grains was cooked with $0.7 \mathrm{~g} \mathrm{Ca}(\mathrm{OH})_{2}$ and $200 \mathrm{~mL}$ distilled water. Nixtamalization time was established as a function of the FI of each sample and measured from the moment the mixture began to boil. After cooking, the samples were steeped for $14 \mathrm{~h}$, after which the cooking solution (nejayote) was discarded. The nixtamal was washed with dap water until all of the pericarp was eliminated and each grain was deheaded. Nixtamal grain without pericarp and pedicel was named "debranned nixtamal." Finally, each sample was boiled with $450 \mathrm{~mL}$ distilled water to allow the grain to swell and open up into a flower shape (become flowered). Flowering time concluded when at least 6 grains $(60 \%)$ of a sample of 10 had flowered.

2.3.2. Industrial Process. In the industrial process, $100 \mathrm{~g}$ of maize was cooked initially with $200 \mathrm{~mL}$ distilled water with $0.7 \mathrm{~g} \mathrm{Ca}(\mathrm{OH})_{2}$. After two min of boiling, $4 \mathrm{~mL}$ of a solution of $50 \% \mathrm{NaOH}(\mathrm{w} / \mathrm{v})$ was added. Nixtamalization of all of the samples lasted $50 \mathrm{~min}$; time was recorded starting from the addition of $\mathrm{NaOH}$. Once the nixtamalization process ended, the cooking solution (nejayote) was discarded and the cooked grain (nixtamal) was washed until all of the pedicel and the pericarp were eliminated. The debranned nixtamal was then bleached in $200 \mathrm{~mL}$ distilled water at $70^{\circ} \mathrm{C}$ with $6 \mathrm{~g} \mathrm{Na}_{2} \mathrm{~S}_{2} \mathrm{O}_{5}$. When the mixture temperature cooled to $50^{\circ} \mathrm{C}$, $2 \mathrm{~mL} \mathrm{CH}_{3} \mathrm{COOH}$ was added and the mixture was left to stand for $20 \mathrm{~h}$. After this period, the solution was discarded and the bleached precooked grain was flowered in the same manner as in the TP.

\subsection{Quality Variables of Debranned Nixtamal, Bleached Pre- cooked Grain, and Flowered Grains}

2.4.1. Debranned Nixtamal (DN). DN yield was expressed as $\mathrm{kg}$ of $\mathrm{DN}$ obtained from each $\mathrm{kg}$ of unprocessed maize $[1,3]$. DN expansion volume was calculated as the difference between the final $\mathrm{DN}$ volume and the initial grain volume [3]. DN moisture content was determined with method 4419.01 of AACC International [12]. DN hardness, expressed as maximum puncture force, was obtained from 10 grains with a texturometer Brookfield ${ }^{\circledR}$ Model CT3 (Middleboro, MA, USA) equipped with a needle $1.0 \mathrm{~mm}$ in diameter and $45 \mathrm{~mm}$ long. The assessment parameters were speed $2 \mathrm{~mm} / \mathrm{s}$ and $5 \mathrm{~mm}$ penetration. Luminosity $\left(L^{*}\right)$ of the $\mathrm{DN}$ was measured with the colorimeter Hunter Lab MiniScan XE Plus Model 45/0-L (Reston, VA, USA), with a D/65 illuminant and a $10^{\circ}$ angle, following the same procedure used in the measurement of grain luminosity [15]. Loss of dry matter in the cooking solution (nejayote) due to the nixtamalization process was quantified as the residual dry matter left after each solution evaporated.

All variables were analyzed in duplicate for each batch, except for hardness where 10 grains were analyzed.

2.4.2. Bleached Precooked Grain. Bleached precooked grain yield was reported as $\mathrm{kg}$ of bleached precooked grain per $\mathrm{kg}$ of unprocessed maize. Precooked grain expansion volume 
was calculated as the difference between the final volume of precooked grain and initial grain volume. Moisture content was determined with AACC International Method 44-19.01 [12], and precooked grain luminosity and hardness were determined as described above. Loss of dry matter in the solution of the bleaching process was quantified as the residual dry matter left after each solution evaporated.

All variables were analyzed in duplicate for each batch, except for hardness where 10 grains were analyzed.

2.4.3. Flowered Grains. Flowered grain yield was reported as $\mathrm{kg}$ of flowered grain per $\mathrm{kg}$ of unprocessed maize [1, $3]$. Flowered grain expansion volume was calculated as the difference between the final volume of flowered grain and initial grain volume. Moisture content was determined with AACC International method 44-19.01 [12]. Flowered grain hardness and luminosity were determined as described above. Flowering time was defined as the time necessary for at least $60 \%$ of the grains to take on the appearance of a flower [4]. Flowered grain solution viscosity was measured using the viscometer Brookfield DV-II+Pro ${ }^{\circledR}$ (Middleboro, MA, USA) in $200 \mathrm{~mL}$ solution samples at $25^{\circ} \mathrm{C}$ with a stainless steel cylindrical probe LV-1 (61) at a rotation rate of $50 \mathrm{rpm}$. Measurements were taken in duplicate when the reading stabilized.

All variables were analyzed in duplicate for each batch, except for hardness where 10 grains were analyzed.

2.5. Pasting Properties of Grain, Debranned Nixtamal, Bleached Precooked Grain, and Flowered Grain. At the end of each stage of each process, a sample of $50 \mathrm{~g}$ of debranned nixtamal, bleached precooked grain, and flowered grain was taken and subjected to a dehydration process in an oven for $24 \mathrm{~h}$ at $40^{\circ} \mathrm{C}$. Then, a $15 \mathrm{~g}$ sample was ground in a FOSS Cyclotec 1093 Mill (Zhanye Rd, SIP, Suzhou, China) and passed through a US \#60 mesh. Pasting properties of the whole grain, debranned nixtamal, bleached precooked grain, and flowered grain were obtained with a method based on Narváez-González et al. [16] using a Rapid Visco Analyzer (RVA) (Super 4, Newport Scientific Pty Ltd., Sydney, Australia). In aluminum receptacles, $4 \mathrm{~g}$ of flour was mixed with $24 \mathrm{~mL}$ distilled water (adjusting moisture to $14 \%$ ). The mixture was heated from 50 to $90^{\circ} \mathrm{C}$ in $9.0 \mathrm{~min}$, and cooking was maintained at $90^{\circ} \mathrm{C}$ for $5 \mathrm{~min}$. Finally, it was cooled to $50^{\circ} \mathrm{C}$ in $9.0 \mathrm{~min}$. Shaking speed was $160 \mathrm{rpm}$.

All variables were analyzed in duplicate for each batch.

2.6. Chemical Composition of Grain, Debranned Nixtamal, Bleached Precooked Grain, and Flowered Grain. Crude protein content was determined by Kjeldahl nitrogen analysis $(N \times 6.25)$ (AACCI Method 46-16.01), crude fat by extraction with petroleum ether (AACCI Method 30-25.01), ash by dry combustion (AACCI Method 08-01.01), and crude fiber by acid-alkaline digestion (AACCI Method 32-10-01) [12]. Carbohydrates were determined by difference $(100-\%$ crude protein $-\%$ crude fat $-\%$ ash $-\%$ crude fiber). Total starch content was determined with a Megazyme total starch assay kit (Megazyme, Bray, Ireland) based on AACCI Approved
Method 76-13.01 [12], and amylose was determined according to the method reported by Hoover and Ratnayake [17]. All variables were analyzed in duplicate for each batch, except for starch and amylose content where three analytical replicates were run.

2.7. Statistical Analysis. The experimental design was completely randomized for the physical and chemical variables of the grain and factorial $4 \times 2$ for quality variables, proximate composition, and pasting properties of processed grains. The results were analyzed with an analysis of variance, comparison of means (Tukey, $p<0.05$ ), and simple Pearson correlation in SAS software for Windows, version 9.0.

\section{Results and Discussion}

3.1. Physical Grain Characteristics and Chemical Composition. Physical grain characteristics and chemical components were significantly different $(p \leq 0.05)$ among the assessed landraces (Tables 1 and 2).

Cacahuacintle had the lowest test weight (TW), large and very soft $(\mathrm{FI}=100)$ grains, and the highest proportion of floury endosperm $(\mathrm{FE}=80.4 \%)$. Similar results with collections of the same landrace were reported by other authors [1, 2, 5, 6]. Ancho and Bofo-Harinoso de Ocho were characterized by low TW and very soft grains. In contrast, some landraces, such as Tuxpeño and Cónico (commonly used for making tortillas) had high TW $\left(>74 \mathrm{~kg} \mathrm{hL}^{-1}\right)$, intermediate to soft hardness according to norm NMX-FF034/1-SCFI-2002 [13], small grains, and larger proportion of vitreous than of floury endosperm. It was also observed that grains from landraces little used to make pozole (Cónico, Tuxpeño, and Bolita) had high contents $(>8.0 \%)$ of fibrous structures (pedicel and pericarp) resulting in a higher content of crude fiber (Tables 1 and 2). Grains of the landrace BofoHarinoso de Ocho had the highest percentage of germ (13.7\%) and the highest lipid content (Tables 1 and 2). According to Figueroa et al. [1], this has highly important technological implications because these lipids can participate in the formation of amylose-lipid complexes during boiling in TP, increase starch granule stability at collapse, and affect the properties of the end product. Pericarp thickness varied between 102 and $111 \mu \mathrm{m}$. These values coincided with those reported by Wolf et al. [14] but were higher than those reported by Narváez-González et al. [18]. Differences are attributed to the method used to determine pericarp thickness. Ancho and Cacahuacintle had the whitest grains $\left(L^{*}>76\right)$ (Table 1$)$.

Contents of crude fat, crude protein, and ash were close to the ranges reported by Narváez-González et al. [18] for a large number of Mexican landraces. Tabloncillo had the highest content of protein and ash (Table 2). The carbohydrate content was higher in Cacahuacintle (Table 2); similar results were reported by Figueroa et al. [5]. Landraces with very soft grains (Cacahuacintle and Bofo-Harinoso de Ocho) had the highest total starch content and the lowest content of amylose according to the trend reported by Dombrink-Kurtzman and Knutson [19]. 


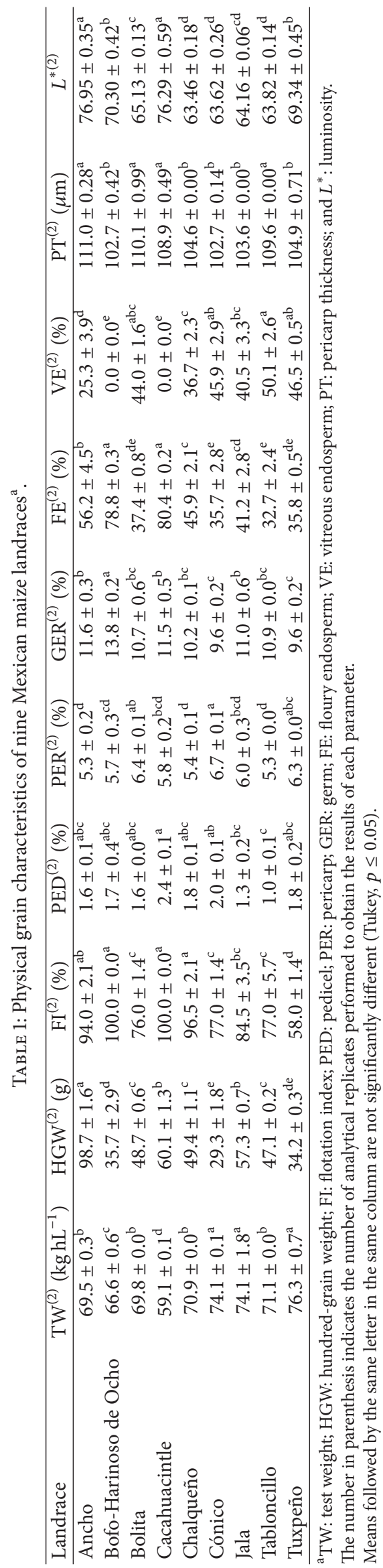




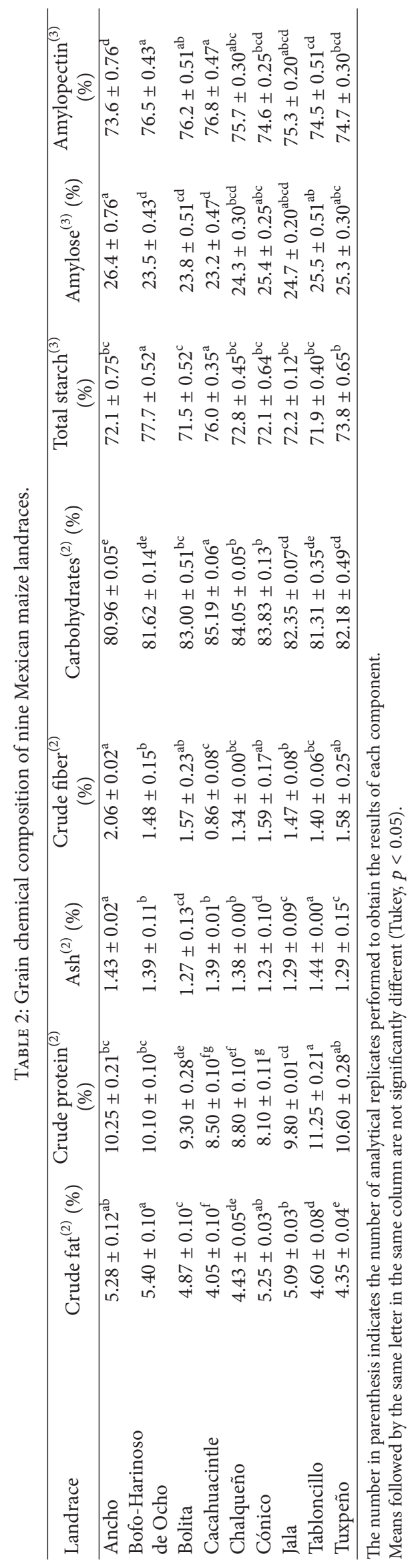


3.2. Effect of Process on Quality Variables and Chemical Components of Debranned Nixtamal, Bleached Precooked Grain, and Flowered Grain. Quality variables and chemical components of debranned nixtamal, bleached precooked grain, and flowered grain were significantly different $(p \leq$ 0.05 ) between treatments due to the effects of different processes, landrace type, and process by landrace interaction. The exceptions were the ash content in debranned nixtamal obtained by industrial process (Table 3), dry matter loss due to bleaching process (DMLB) (Table 4), and crude fiber content in flowered grains (Table 5).

TP resulted in higher debranned nixtamal yield, larger expansion volume, and higher moisture content, as well as softer debranned nixtamal relative to the debranned nixtamal produced by the IP (Table 3). Those differences were mainly due to processing time. Nixtamalization and steeping time in TP $(\sim 15 \mathrm{~h})$ allowed starch granules to hydrate more, while in the IP water absorption occurred only during nixtamalization $(<1 \mathrm{~h})$. Cacahuacintle in TP as well as Cacahuacintle and Bofo-Harinoso de Ocho in IP had the highest yield, expansion volume, and moisture content in the debranned nixtamal. TW, FE, and VE were the physical variables that most correlated with the variables measured in debranned nixtamal. Higher-density grains (high TW) produced harder (TP: $r=0.62, p \leq 0.01$; IP: $r=0.72, p \leq 0.01$ ) debranned nixtamal with low moisture content (TP: $r=-0.60, p \leq 0.01$; IP: $r=-0.68, p<0.01)$. In addition, in both processes grains with high $\mathrm{FE}$ content had debranned nixtamal with higher expansion volume (TP: $r=0.59, p \leq 0.05$; IP: $r=0.47, p \leq$ 0.05 ) and higher moisture content (TP: $r=0.54, p \leq 0.05$; IP: $r=0.88, p<0.01$ ) and were therefore softer (TP: $r=-0.75$, $p \leq 0.01$; IP: $r=-0.77, p<0.01$ ). These results could be related to the degree of compaction of the starch granules and the protein matrix that surrounds these granules. NarváezGonzález et al. [16] found that the grains with a larger proportion of FE have many intragranular spaces and a sparse protein matrix, facilitating penetration and diffusion of water into the grain and thus increasing water absorption of the grain. In TP, larger grains also favored high expansion volumes $(r=0.63, p<0.01)$.

The higher crude fiber content decreased the yield of debranned nixtamal due to a higher content of cellulose, hemicellulose, and lignin in pericarp which does not allow the rapid diffusion of water into the grain. Additionally, landraces with higher starch content resulted in a higher expansion volume, higher moisture content, and softer debranned nixtamal grains in both processes.

Loss of dry matter caused by the nixtamalization process was higher in IP than in TP (Table 3). From a nutritional and environmental standpoint, high losses of dry matter mean loss of nutrients, mainly crude fat and crude fiber (Tables 2 and 3), and more pollution from the effluents. However, in the nixtamalization process for pozole preparation, elimination of the fibrous part of the grain (pedicel and pericarp) is essential for the subsequent stages (bleaching and flowering in IP and flowering in TP). In IP, high losses of dry matter and the lowest content of crude fiber in debranned nixtamal indicate the effectiveness of the $\mathrm{Ca}(\mathrm{OH})_{2}+\mathrm{NaOH}$ solution in hydrolysis of these structures, as Singh et al. [20] have reported. Similar values for these variables were reported by Figueroa et al. [1] and Vázquez-Carrillo and Santiago-Ramos [3].

The color of the debranned nixtamal depends on the color of the layer of aleurone that remains on the grain after nixtamalization, during which $\mathrm{Ca}(\mathrm{OH})_{2}$ and $\mathrm{NaOH}$ give it a yellowish tone. In some landraces, color of the debranned nixtamal could also be greatly influenced by the color of the endosperm. The debranned nixtamal obtained by TP had greater luminosity (was whiter) than that obtained by IP (Figure 2), which were results similar to those reported by Vázquez-Carrillo and Santiago-Ramos [3].

According to González et al. [21], undesirable nixtamal color is due to the lignin fraction that easily oxidizes in an alkaline medium. However, there is little information on this matter. Because of the yellowish tone of the debranned nixtamal obtained by IP, it is necessary to use bleaching agents because most consumers, especially in Central Mexico, prefer white grains.

In Mexico, consumption of bleached precooked grain has increased significantly because of the savings in processing time. The industries that produce precooked grain prefer high-yielding maize types with large expansion volumes and very white grains. To obtain high yields and large expansion volumes, it is necessary that, during the $20 \mathrm{~h}$ process, grains absorb as much water as possible. Precooked grain yield, expansion volume, and moisture content increased relative to the values obtained in debranned nixtamal, because of the $20 \mathrm{~h}$ the debranned nixtamal stands in the $\mathrm{CH}_{3} \mathrm{COOH}$ $\mathrm{Na}_{2} \mathrm{~S}_{2} \mathrm{O}_{5}$ solution. The highest yield and expansion volume of precooked grain were obtained with the landrace Cacahuacintle, followed by Bofo-Harinoso de Ocho, which absorbed a greater quantity of water. The lowest values were obtained with Tabloncillo (Table 4). TW, FI, and FE correlated highly with the evaluated variables, revealing that diffusion and absorption of water during bleaching were also influenced by density, hardness, and degree of compaction of the starch granules. Therefore, the highest yields and expansion volumes were obtained with grains with low TW, high FI, and high FE. Similar values of moisture content and hardness of precooked grain were found in the commercial samples of precooked grains (Table 4). Losses of dry matter during this stage were 3.1-3.5\%; these percentages were lower than those reported by Vázquez-Carrillo and Santiago-Ramos [3] for the Cacahuacintle landrace (5.2\%).

With respect to grain chemical components, landraces with higher starch content in grain had higher yield $(r=0.83$, $p \leq 0.01)$, higher expansion volume $(r=0.63, p \leq 0.01)$, and higher moisture content $(r=0.82, p \leq 0.01)$ as well as $\operatorname{softer}(r=-0.68, p \leq 0.01)$ bleached precooked grains. Additionally, debranned nixtamal with lower content of crude fiber allowed obtaining high yield of bleached precooked grains.

After bleaching, all the maize landraces increased in luminosity (Figure 2). Bofo-Harinoso de Ocho and Ancho landraces had the highest luminosity. The high luminosity values of the commercial samples may be due to a longer bleaching period $(>20 \mathrm{~h})$ or to the use of a higher concentration of sodium metabisulfite. 


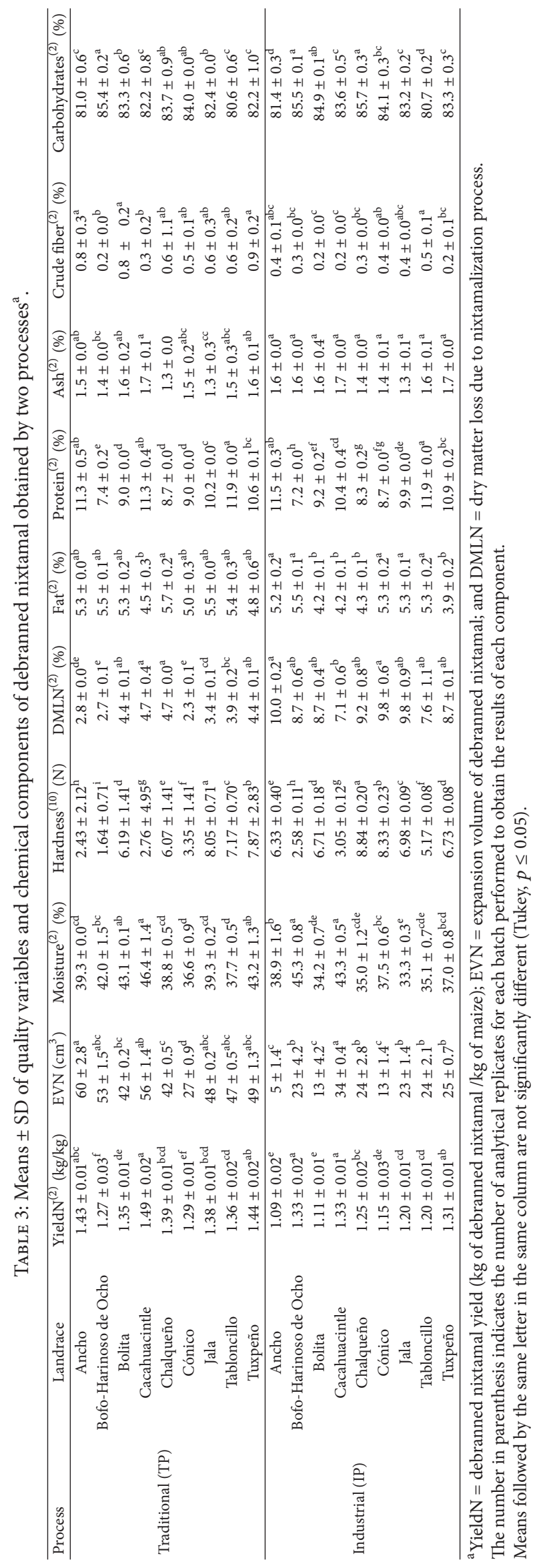




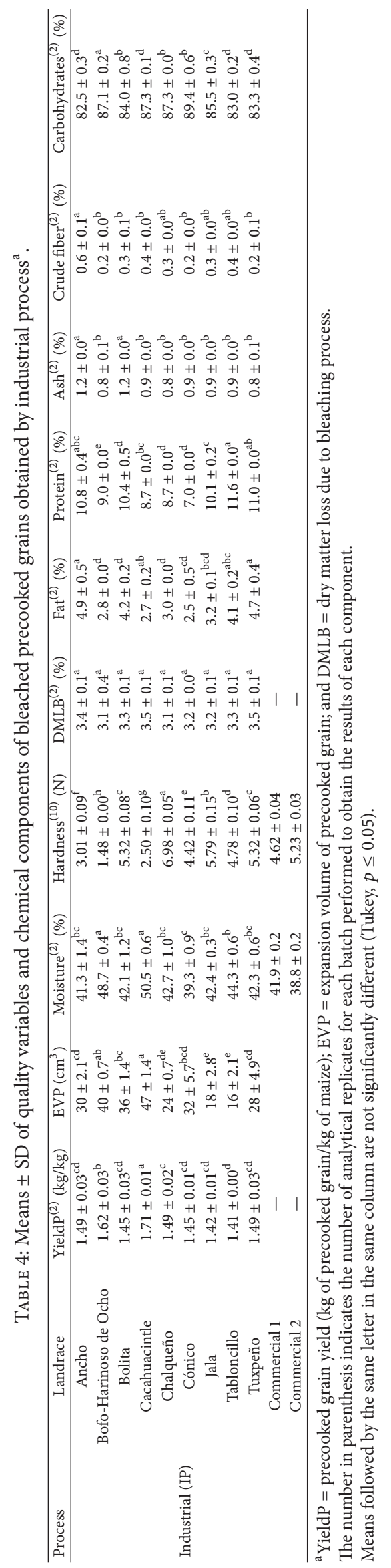




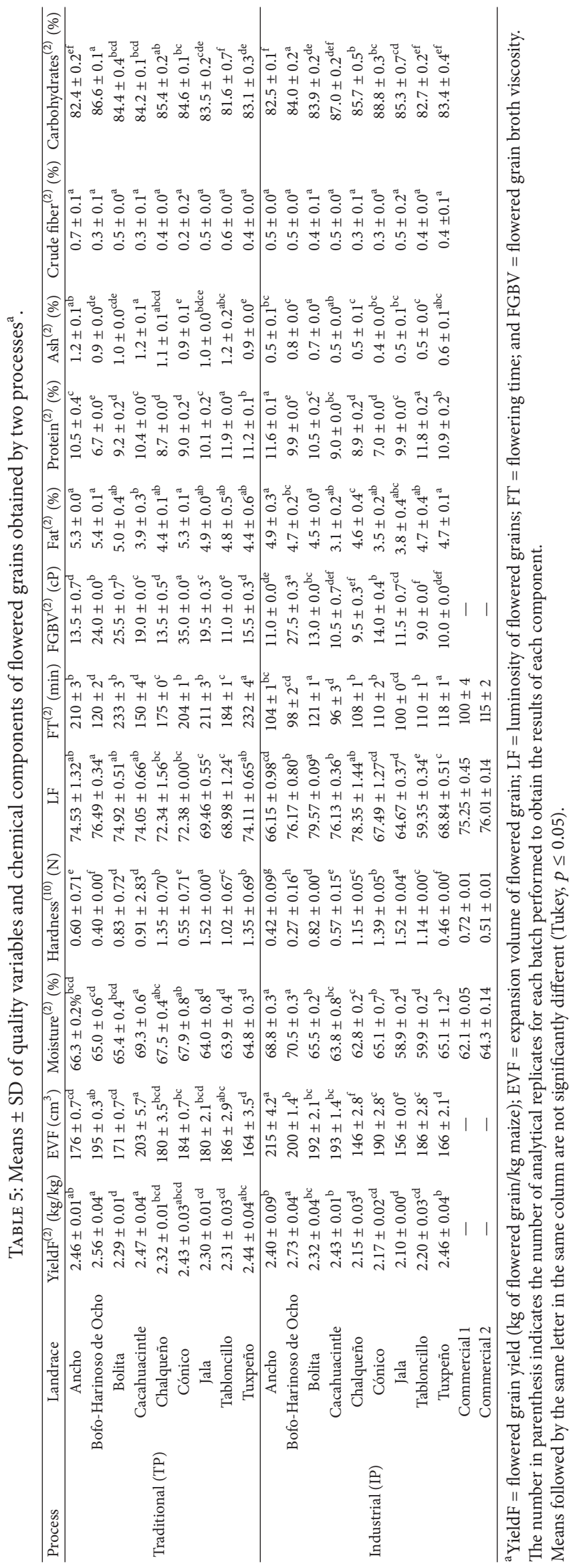




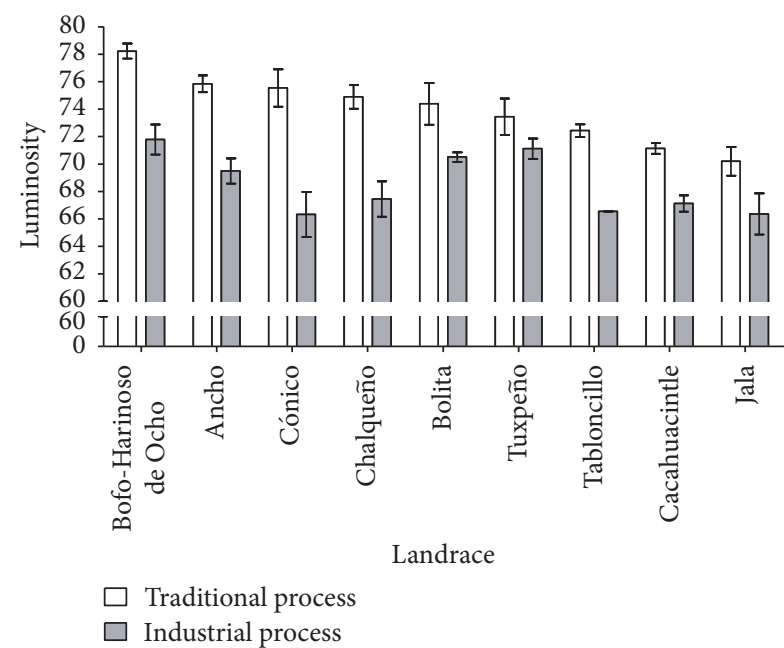

(a)

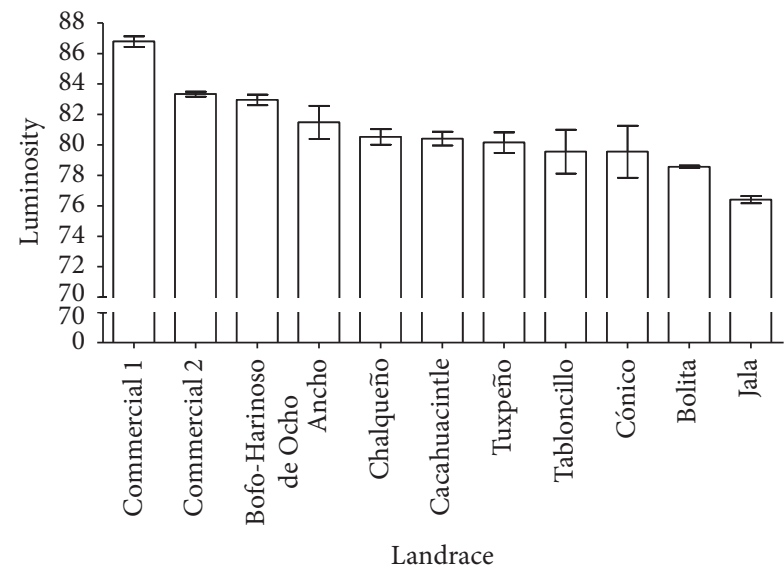

(b)

FIGURE 2: Luminosity of debranned nixtamal (a) and bleached precooked grain (b) from nine maize landraces.

The last stage of making pozole in TP involves boiling the debranned nixtamal and, in IP, boiling bleached precooked grain. This stage is known as flowering (Figure 1). The most critical variable in this stage is the flowering time, which requires a lot of energy. Short flowering times as well as high expansion volumes and yields are desirable. In both processes, Cacahuacintle and Bofo-Harinoso de Ocho require less time to flower (Table 5). Both landraces have rounded grains; Bonifacio Vázquez et al. [4] reported that roundedglobose grains are those that require shorter flowering times. In TP, flowering time was $120 \mathrm{~min}$ for Bofo-Harinoso de Ocho and up to $233 \mathrm{~min}$ for the Bolita landrace, similar to the values reported by other authors $[1,3,4,6]$. Debranned nixtamal hardness significantly affected flowering time: the harder the debranned nixtamal grain, the longer the flowering time $(r=$ $0.74, p \leq 0.01)$.

In IP, flowering time was shorter (96-121 min) than in TP (Table 5). Moisture content and precooked grain hardness affected flowering time. Those samples in which the bleached precooked grain had high moisture content and soft grains required less time to flower $(r=-0.54$ and $r=0.71, p<$ 0.01 , resp.). In both processes, Bofo-Harinoso de Ocho and Cacahuacintle had the highest yield, high expansion volume, and high moisture content of flowered grain. In contrast, Bolita in TP and Jala in IP had the lowest yield (Table 5). Figueroa et al. [5] showed that the starch granule transforms into a sponge-like structure that permits absorption of a large amount of water, explaining the high expansion volume and moisture content of the flowered grain. This behavior is more accentuated in soft-grained landraces or in those with a high content of floury endosperm such as Cacahuacintle and BofoHarinoso de Ocho.

There was a difference of $0.27 \mathrm{~kg}$ in the TP and $0.63 \mathrm{~kg}$ in the IP among landraces with the highest and lowest flowered grain yield. With Bofo-Harinoso de Ocho landrace the best yield and expansion volume of flowered grain was obtained, and, from the economic point of view, the use of this landrace could represent higher income for both restaurants that sell pozole every day and for industries that produce large volumes of "instant pozole."

A soft-textured flowered grain is easy to chew and more palatable for the end consumer. In both processes, BofoHarinoso de Ocho had the softest flowered grains while Jala had the hardest. Viscosity of the flowered grain broth was higher in TP than in IP because of the longer flowering time. Our results showed that in both processes grain lipid content correlates highly with flowering broth viscosity (TP: $r=0.66$ and IP: $r=0.70, p<0.01)$.

Flowered grain luminosity decreased 0.0 to $4.8 \%$ relative to TP debranned nixtamal and 0.0 to $25.4 \%$ relative to bleached grain of IP, as was also reported by Figueroa et al. [1] and Vázquez-Carrillo and Santiago-Ramos [3]. The greater opacity of flowered grains is due to the loss of crystallinity and organized structure of the starch granules.

3.3. Effect of Process on Pasting Properties of Grain, Debranned Nixtamal, Precooked Grain, and Flowered Grain. Regarding pasting properties, grains of the soft and light (low TW) maize landraces had low pasting temperature and high peak viscosity and setback (Table 6). Narváez-González et al. [18] found that the maize landraces that developed high peak viscosity were softer, having a lower degree of compaction and larger starch granules, which facilitates diffusion of water into the grain and results in greater swelling.

In TP, pasting temperature of debranned nixtamal increased between 0.63 and $4.85^{\circ} \mathrm{C}$ relative to that of the grain (Table 6) as a consequence of steeping, during which the annealing phenomenon occurs [1]. In addition, a significant increase in the peak viscosity and setback of the debranned nixtamal starch was observed with all the landraces. This increase is attributed to an increase in granule rigidity and resistance to shear stress. Similar results for TP were reported by Figueroa et al. [5]. The debranned nixtamal from IP did not undergo a significant increase in pasting temperature 


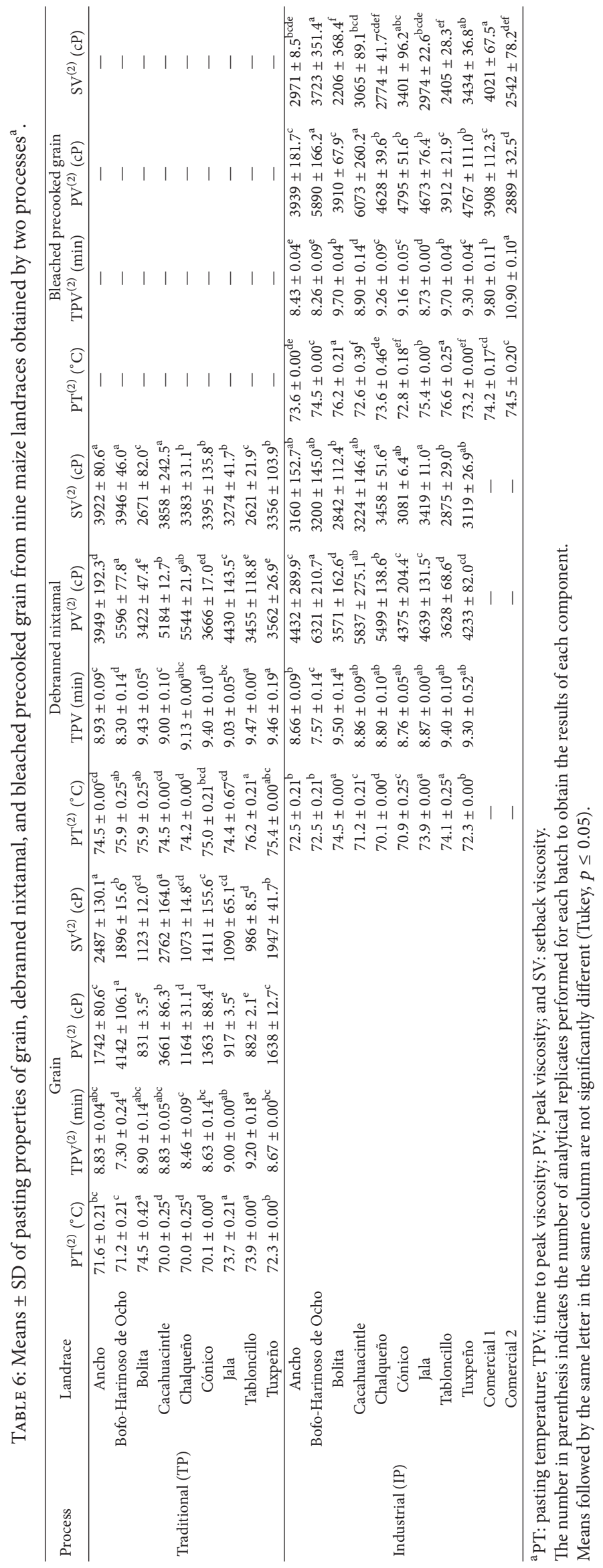




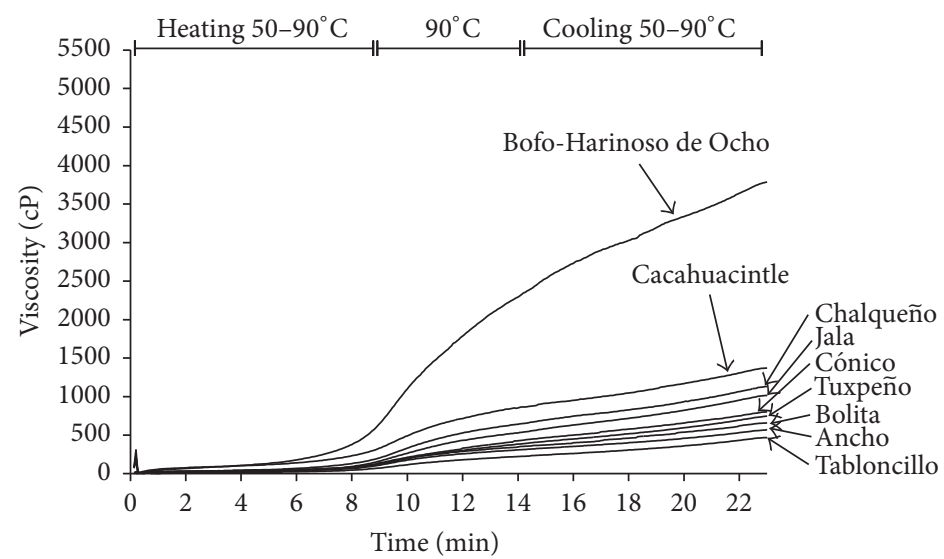

(a)

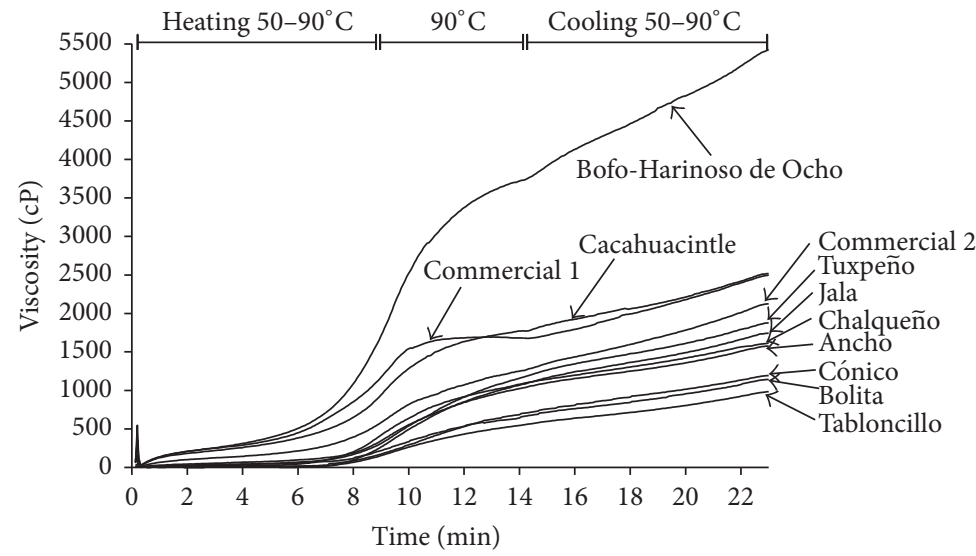

(b)

FIGURE 3: Viscoamylographic curves of flowered grains from nine maize landraces and commercial samples. (a) Traditional process. (b) Industrial process.

because there is no steeping in this process, so the annealing phenomenon could not occur. Nevertheless, significant increases were observed in peak viscosity and setback. Nor Nadiha et al. [22] and Nyakabau et al. [23] reported that the increase in starch viscosity of the maize treated with $\mathrm{NaOH}$ is related to the absence of the protein matrix that surrounds the starch granules. Most of these proteins could have been hydrolyzed during cooking in the $\mathrm{Ca}(\mathrm{OH})_{2}+\mathrm{NaOH}$ solution. The decrease or elimination of the protein matrix allowed the starch granule to swell more quickly. This was confirmed by the significant correlation of protein content in the debranned nixtamal with peak viscosity $(r=-0.57, p \leq 0.01)$ and time to peak viscosity $(r=0.66, p \leq 0.01)$. In other words, samples with a higher content of protein in the debranned nixtamal took longer to reach low peak viscosity (Tables 3 and 6). Lai et al. [24] attributed the increase of setback in starch treated with $\mathrm{NaOH}$ to a depolymerization of the starch into chains with a degree of polymerization in the interval of 15 to 20, as those chains have been shown to have a greater tendency to aggregate and crystalize. However, in pozole preparation more research is needed to understand the effect of $\mathrm{NaOH}$ cooking on the maize starch and other components.
During the bleaching stage of IP, it was observed that the starch underwent a similar effect like the annealing process occurring in TP during nixtamal steeping. This was an increase in pasting temperature, peak viscosity, and time to peak viscosity of precooked grain relative to debranned nixtamal (Table 6). The increase in pasting temperature was less (0.90-3.43) than that in TP during steeping, and these results could be due to the lower temperature at which bleaching is carried out $\left(<50^{\circ} \mathrm{C}\right)$. According to Jayakody and Hoover [25], the effect of annealing on the starch structure is more pronounced if the annealing temperature is very close to the gelatinization onset temperature. The increase in the peak viscosity of most of the landraces is due to the increase in starch granule rigidity caused by annealing.

The pasting properties of the flowered grain decreased drastically (Figure 3) because the semicrystalline starch structure was completely lost during flowering.

The decrease is greater in TP because the starch granules were boiled for a longer time. Figure 3 shows how viscosity increases from 80 to $90^{\circ} \mathrm{C}$ and rises during cooling; in both processes Bofo-Harinoso de Ocho showed the highest final 
viscosity. This behavior could be related to the amyloseamylopectin ratio and amylose-lipid complexes present in the flowered grain.

According to Tang and Copeland [26] the increase in final viscosity is indicative of the presence of amylose-lipid complexes, as Figueroa et al. [1] demonstrated in the flowered grain obtained by TP. Our results show that the race with the highest content of lipids (5.40\%) in the grain (Bofo-Harinoso de Ocho) had the highest final viscosity of flowered grain, while the races with lower lipid contents had low final viscosity in both processes (Figure 3). Additionally, final viscosity of flowered grains by TP was strongly correlated with a high carbohydrates content $(r=0.75, p \leq 0.01)$ and low protein content in flowered grains $(r=-0.78, p \leq 0.01)$. However, more research on this aspect is needed.

\section{Conclusions}

In TP water absorption occurred during nixtamalization and steeping time $(\sim 15 \mathrm{~h})$, while in the IP this event occurred during bleaching. In both processes, the technological variables of debranned nixtamal, bleached precooked grain, and flowered grain are closely related to the grain physical properties. Very soft $(\mathrm{FI}=100)$ grains, with low test weight $\left(<67 \mathrm{~kg} \mathrm{hL}^{-1}\right)$, high proportion of floury endosperm (FE $>78.0 \%)$, and high peak viscosity $(>3661 \mathrm{cP})$, had higher water absorption and starch swelling during nixtamalization and bleaching stages, producing higher yield and volume of debranned nixtamal and bleached precooked grains. An increase in pasting temperature, peak viscosity, and time to peak viscosity was observed in TP during steeping and in IP during bleaching. IP decreased processing times as well as flowering time; however it produces higher dry matter losses than TP. Based on technological variables of flowered grains, the potential of landraces for this purpose was as follows: Bofo-Harinoso de Ocho $>$ Cacahuacintle $>$ Ancho $>$ Cónico $\sim$ Tabloncillo $\sim$ Chalqueño $\sim$ Jala $>$ Tuxpeño $\sim$ Bolita.

\section{Additional Points}

Practical Applications. This study could be used for all the stakeholders on the whole value chain of pozole market. Farmers can prove that the landraces they grow have better quality of processed grain for pozole compared to hybrids or varieties mainly used for tortilla production. In the pozole market, farmers can continue to sell their grain at a price per kilogram two or three times higher than hybrids; so they could generate more income and prevent the extinction of some landraces through "conservation in situ" or "conservation through use." Processors can identify the best landrace or characteristics needed to obtain higher yield and very white bleached precooked grains and add value to grain and sell it in different forms, either as deheaded grain, as bleached precooked grain, or as instant pozole. Finally, consumers can appreciate these landraces for their culinary features, such as color, texture, and taste, so we can understand why the pozole prepared with Cacahuacintle and Ancho is already considered by consumers to be premium-quality.

\section{Competing Interests}

The authors declare that there is no conflict of interests regarding the publication of this paper.

\section{Acknowledgments}

Authors are grateful to Coordinadora Nacional de las Fundaciones Produce, A. C. (COFUPRO), for the financial support to conduct this research, as well as Dr. Noel Orlando Gómez Montiel and Dr. J. Arahón Hernández Guzmán for providing us with the germplasm evaluated.

\section{References}

[1] J. D. D. Figueroa, J. J. V. Véles Medina, M. A. Hernández Landaverde et al., "Effect of annealing from traditional nixtamalisation process on the microstructural, thermal, and rheological properties of starch and quality of pozole," Journal of Cereal Science, vol. 58, no. 3, pp. 457-464, 2013.

[2] M. G. Vázquez-Carrillo, D. Santiago-Ramos, Y. SalinasMoreno, and J. E. Cervantes-Martínez, "El pozole. Situación actual y calidad nutricional," in Los Alimentos en México y su Relación con la Salud, M. Aguilera Ortiz, R. Reynoso Camacho, C. A. Gómez Aldapa, R. M. Uresti-Marín, and J. A. Ramírez de León, Eds., pp. 113-133, Plaza y Valdés, Veracruz, Mexico, 2014.

[3] M. G. Vázquez-Carrillo and D. Santiago-Ramos, "Physicochemical characteristics and pozole quality of Cacahuacintle maize processed by three methods," Revista Fitotecnia Mexicana, vol. 36, supplement 3, pp. 357-366, 2013.

[4] E. I. Bonifacio Vázquez, Y. Salinas Moreno, A. Ramos Rodríguez, and A. Carrillo Ocampo, "Quality for pozole preparation in accessions of the Cacahuacintle maize," Revista Fitotecnia Mexicana, vol. 28, no. 3, pp. 253-260, 2005.

[5] J. D. C. Figueroa, J. J. Véles-Medina, E. M. Tolentino-López et al., "Effect of traditional nixtamalization process on starch annealing and the relation to pozole quality," Journal of Food Process Engineering, vol. 36, no. 5, pp. 704-714, 2013.

[6] C. A. Hernández Galeno, Y. Salinas Moreno, P. Antonio Lopéz, A. Santacruz Varela, F. Castillo González, and T. Corona Torres, "Quality for pozole in Cacahuacintle maize populations from High Valleys of Puebla," Revista Mexicana de Ciencias Agrícolas, vol. 5, no. 4, pp. 703-716, 2014.

[7] J. Ron Parra, J. J. Sánchez González, A. A. Jiménez Cordero et al., "Maíces nativos del occidente de México I. Colectas 2004," Scientia-CUCBA, vol. 8, no. 1, pp. 1-139, 2006.

[8] A. Keleman and J. Hellin, "Specialty maize varieties in Mexico: a case study in market-driven agro-biodiversity conservation," Journal of Latin American Geography, vol. 8, no. 2, pp. 147-174, 2009.

[9] National Commission for Knowledge and Use of Biodiversity (CONABIO), Tabla Descriptiva de Razas de Maíz de México, CONABIO, Mexico City, Mexico, 2012, http://www.biodiversidad.gob.mx/genes/pdf/proyecto/Anexo6_ReunionesTalleres/ Tabla\%20razas_marzo\%202010.pdf.

[10] J. Hellin, A. Keleman, D. López, L. Donnet, and D. Flores, “The importance of niche markets. A case study of blue and pozolemaking maize in México," Revista Fitotecnia Mexicana, vol. 36, supplement 3, pp. 315-328, 2013. 
[11] A. Keleman, H. García Rañó, and J. Hellin, "Maize diversity, poverty, and market access: lessons from Mexico," Development in Practice, vol. 19, no. 2, pp. 187-199, 2009.

[12] AACC International, Approved Methods of Analysis, Method 08-01.01, Ash-Basic method, 1961, Method 30-25.01, Crude fat in wheat, corn, and soy flour, feeds, and mixed feeds, 1961, Method 44-19.01, Moisture-Air-oven method, drying at $135^{\circ}, 1961$, Method 46-16.01, Crude protein-Improved Kjeldahl method, copper-titanium dioxide catalyst modification, 1988, Method 55-10.01, Test Weight per Bushel, Method 76-13.01, Total starch assay procedure (Megazyme amyloglucosidase/ $\alpha$-amylase method), 11th Ed, AACCI, St. Paul, Minn, USA, 1995.

[13] Secretaría de Economía, "Non industrialized food products for human consumption-cereals-part 1: white corn for alkaline process of corn tortillas and nixtamalized corn productsspecifications and test methods," SE NMX-FF-034/1-SCFI2002, General Department of Standards, Mexico City, Mexico, 2002.

[14] M. J. Wolf, I. M. Cull, J. L. Helm, and M. S. Zuber, "Measuring thickness of excised mature corn pericarp," Agronomy Journal, vol. 61, no. 5, pp. 777-779, 1969.

[15] C. D. Floyd, L. W. Rooney, and A. J. Bockholt, "Measuring desirable and undesirable color in white and yellow food corn," Cereal Chemistry, vol. 72, no. 5, pp. 488-490, 1995.

[16] E. D. Narváez-González, J. D. D. Figueroa-Cárdenas, S. Taba, and F. R. Sánchez, "Kernel microstructure of Latin American races of maize and their thermal and rheological properties," Cereal Chemistry, vol. 83, no. 6, pp. 605-610, 2006.

[17] R. Hoover and W. S. Ratnayake, "Determination of total amylose content of starch," in Current Protocols in Food Analytical Chemistry, UNIT E2.3, pp. E2.3:1-E2.3.5, 2001.

[18] E. D. Narváez-González, J. D. D. Figueroa-Cárdenas, S. Taba, E. Castaño Tostado, R. Á. Martínez Peniche, and F. Rincón Sánchez, "Relationships between the microstructure, physical features, and chemical composition of different maize accessions from Latin America," Cereal Chemistry, vol. 83, no. 6, pp. 595-604, 2006.

[19] M. A. Dombrink-Kurtzman and C. A. Knutson, "A study of maize endosperm hardness in relation to amylose content and susceptibility to damage," Cereal Chemistry, vol. 74, no. 6, pp. 776-780, 1997.

[20] S. K. Singh, N. Singh, L. Du, and S. R. Eckhoff, "Effect of sodium hydroxide, calcium hydroxide, and potassium hydroxide on debranning of corn," Cereal Chemistry, vol. 74, no. 3, pp. 254257, 1997.

[21] R. González, E. Reguera, L. Mendoza, J. M. Figueroa, and F. Sánchez-Sinencio, "Physicochemical changes in the hull of corn grains during their alkaline cooking," Journal of Agricultural and Food Chemistry, vol. 52, no. 12, pp. 3831-3837, 2004.

[22] M. Z. Nor Nadiha, A. Fazilah, R. Bhat, and A. A. Karim, "Comparative susceptibilities of sago, potato and corn starches to alkali treatment," Food Chemistry, vol. 121, no. 4, pp. 1053-1059, 2010.

[23] T. Nyakabau, O. C. Wokadala, and M. N. Emmambux, "Effect of steeping additives on tef starch extraction and its quality," Starch, vol. 65, no. 9-10, pp. 738-746, 2013.

[24] L. N. Lai, A. A. Karim, M. H. Norziah, and C. C. Seow, "Effects of $\mathrm{Na}_{2} \mathrm{CO}_{3}$ and $\mathrm{NaOH}$ on pasting properties of selected native cereal starches," Journal of Food Science, vol. 69, no. 4, pp. 249256, 2004.

[25] L. Jayakody and R. Hoover, "Effect of annealing on the molecular structure and physicochemical properties of starches from different botanical origins-a review," Carbohydrate Polymers, vol. 74, no. 3, pp. 691-703, 2008.

[26] M. C. Tang and L. Copeland, "Analysis of complexes between lipids and wheat starch," Carbohydrate Polymers, vol. 67, no. 1, pp. 80-85, 2007. 



Submit your manuscripts at

https://www.hindawi.com
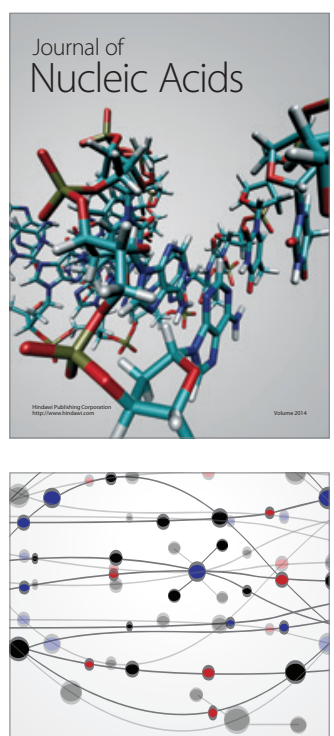

The Scientific World Journal
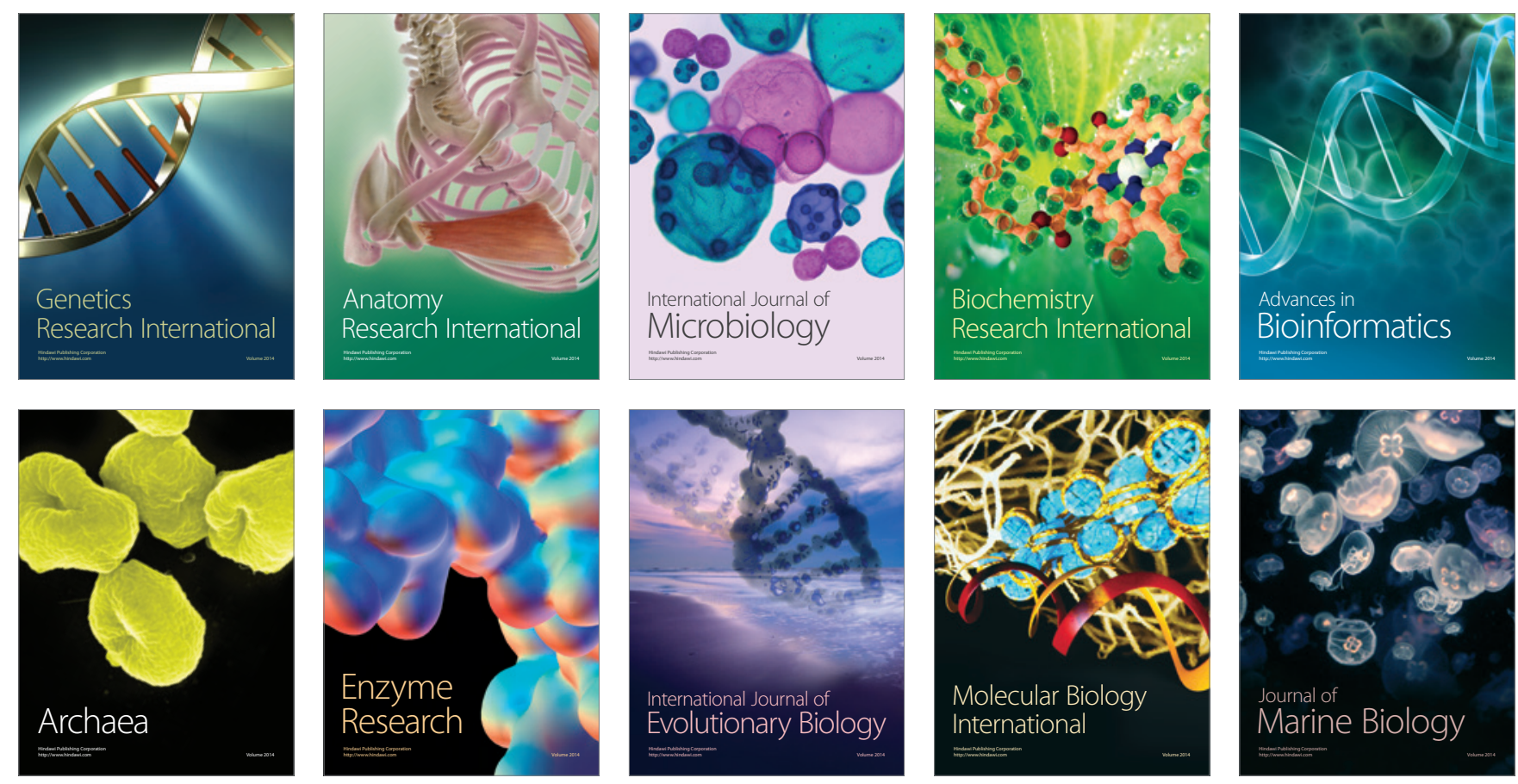